\title{
The effectiveness of lunchbox interventions on improving the foods and beverages packed and consumed by children at centre-based care or school: a systematic review and meta-analysis
}

\author{
Nicole Nathan ${ }^{1,2,3,4,7^{*}}$, Lisa Janssen ${ }^{1}$, Rachel Sutherland ${ }^{1,2,3,4}$, Rebecca Kate Hodder ${ }^{1,2,3,4}$, Charlotte E. L. Evans ${ }^{6}$, \\ Debbie Booth ${ }^{5}$, Sze Lin Yoong ${ }^{1,2,3,4}$, Kathryn Reilly ${ }^{1,2,3,4}$, Meghan Finch 1,2,3,4 and Luke Wolfenden 1,2,3,4
}

\begin{abstract}
Objective: To assess the effectiveness of lunchbox interventions aiming to improve the foods and beverages packed and consumed by children at centre-based care or school; and subsequent impact on children's adiposity.

Methods: Systematic search of nine databases for controlled trials published in English between 1995-January 2017. Where appropriate, data were pooled in a random effects meta-analysis.

Results: Of the 1601 articles identified, ten studies (centre-based care $n=4$, school $n=6$ ) were included of which eight were RCTs. The impact of interventions on the packing of discretionary foods, sugar-sweetened drinks and other core foods was inconsistent. Meta-analysis of four RCTs trials found a moderate increase in provision of vegetables $(\mathrm{SMD}=0$. $4095 \% \mathrm{Cl} 0.16$ to $0.64, p=0.001, I^{2}=82 \%$; equivalent to a mean difference of 0.28 serves) but not fruit. Four studies reported impact on children's dietary intake, one reported no significant effect on consumption of discretionary foods, one reported improvements in the consumption of sugar-sweetened drinks and water, and two reported improvements in consumption of vegetables and fruit. Two studies, that were broader obesity prevention interventions, reported no significant impact on adiposity.
\end{abstract}

Conclusions: There is some evidence that lunchbox interventions are effective in improving the packing of vegetables in children's lunchboxes, however more robust research is required to determine the impact on children's dietary intake and adiposity.

Trial registration: PROSPERO 2016: CRD42016035646.

Keywords: Diet behaviour, Children, Lunchbox, Systematic review, Packed lunch

\section{Introduction}

Children who fail to consume sufficient portions of vegetables and fruit, and overconsume energy-dense, nutrientpoor foods and beverages such as confectionary, potato chips and sugar-sweetened drinks (discretionary foods)

\footnotetext{
* Correspondence: nicole.nathan@hnehealth.nsw.gov.au

${ }^{1}$ Hunter New England Population Health, Hunter New England Local Health District, Newcastle, Australia

${ }^{2}$ School of Medicine and Public Health, The University of Newcastle,

Newcastle, Australia

Full list of author information is available at the end of the article
}

significantly increase their risk of developing future chronic disease and increase their risk of unhealthy weight gain [1-3]. Evidence suggests that a large proportion of children in high-income countries, including the United States (U.S.), United Kingdom (U.K.), and Australia, consume diets that are less than adequate, with a significant proportion of children's energy intake coming from discretionary foods $[4,5]$ and less than $20 \%$ of children meeting recommended vegetable and fruit intake [6-9]. As dietary behaviours established in childhood can track through to adulthood, [10] supporting the establishment 
of healthy dietary habits in childhood has the potential to reduce the burden of obesity and other current and future diet-related disease [11].

Schools and early childhood education and care settings (here after referred to as centre-based care, which include preschools, long day-care services and kindergartens) have been identified as important settings in which to implement population wide interventions to improve child diet and to reduce overweight and obesity, given their continuous and intensive contact with children [12, 13]. Research indicates that children consume between one third [14] to one half [15] of their daily energy intake whilst in schools and centre-based care; providing an opportune time to impact on their dietary intake. Although these settings may provide meals to children, a significant proportion of children in many countries rely on parents and carers to provide all or some of their child's food and beverages for consumption whilst in attendance. For example, in the U.S. and U.K. it is estimated that on any given day $40-50 \%$ of children [16, 17], in both school and centre-based care, bring lunch and/or snacks from home whilst in Australia, Mexico and New Zealand most children bring a packed lunchbox to school [18-20].

Evidence suggests that foods provided by parents for consumption in these settings are not in line with dietary guidelines. For example, Australian observational studies have reported lunchboxes in primary schools have an excess of discretionary foods with the average lunchbox containing 3.1 serves [14]. Similarly, only $1.6 \%$ of primary school children surveyed in the U.K. had packed lunches that met nutritional standards, whilst $52-60 \%$ of lunchboxes contained sweet and savoury discretionary snacks [21]. Conversely the inclusion of core foods i.e. fruits and vegetables, whole grain cereals, lean meats etc. is notably inadequate with one study identifying that only $14 \%$ of the packed lunchboxes of children aged 11-12 years contained a piece of fruit [22]. Studies in centre-based care have found similar patterns. For example, a cross-sectional study conducted in 18 Australian centre-based care services found that; of the 49 children who were observed that brought food from home via a lunchbox, none met the daily dietary recommendations and, children consumed an average of 0.7 serves of discretionary foods [23]. Similarly, a study of 528 pre-school children's lunchboxes in California found that more than $80 \%$ of lunchboxes contained discretionary foods, such as chips and sugar-sweetened drinks, whilst only $16 \%$ included vegetables [24]. Globally, these studies demonstrate a need for effective interventions to support the provision of nutritionally balanced lunchboxes.

Whilst studies have suggested that interventions to improve the nutritional contents of lunchboxes are warranted, [14] very little is known regarding the impact of such interventions on improving the contents of children's lunchboxes. To our knowledge only one systematic review examining the effectiveness of lunchbox interventions has been conducted and included studies published up to the start of 2013 [25]. This narrative review, published in Spanish, included four studies examining the impact of interventions on vegetables and fruit packed in children's lunchboxes and reported a significant increase in the provision of and consumption of vegetables and fruit within lunchboxes [25]. However, the review did not investigate if such lunchbox interventions impacted on the packing or consumption of other foods (i.e. discretionary foods) and beverages, and on students' body mass index (BMI) or adiposity. To address these limitations and guide the development and implementation of effective lunchbox interventions, an updated and comprehensive synthesis of current evidence is needed.

\section{Aims and objectives}

The primary aim of the review was to assess the effectiveness of lunchbox interventions aiming to improve the foods and beverages packed and consumed by children attending centre-based care or school. A secondary aim of the review was to assess the effectiveness of these interventions on child adiposity (e.g. weight or BMI) or waist circumference.

\section{Methods \\ Registration}

The review was prospectively registered with PROSPERO (CRD42016035646) and is reported in accordance to the Preferred Reporting Items for Systematic Reviews and Meta-Analyses (PRISMA) guidelines [26].

\section{Eligibility criteria}

Studies were eligible for inclusion if (i) participants were children aged 2-18 years. Studies with only children aged less than 2 years were ineligible due to the different nutritional requirements and developmental stages of young children; (ii) interventions included any educational, experiential, health promotion and/or family or structural or policy or legislative interventions that targeted food provided from home for child consumption during attendance at school or centre-based care (either explicitly or as part of a broader obesity prevention intervention); (iii) they included parallel comparison groups e.g. randomised controlled trials (RCTs), controlled clinical trials, non-randomised trials; and (iv) reported outcomes included either a change in the number or proportion of serves, portions, or grams of food provided or consumed as measured by direct observation, surveys or weighed food in lunchboxes. Secondary 
outcomes for anthropometry could be measured by BMI, BMI percentile, waist measurements or body composition (e.g. per cent body fat, per cent lean body mass or skin folds). There were no restrictions on length of follow-up time or on publication status. Studies were excluded if interventions were targeting the treatment or management of diagnosed diseases or health problems that impacted on child diet or weight, for example, eating disorders, such as anorexia nervosa or bulimia, or overweight or obesity.

\section{Information sources and search strategy}

A comprehensive search strategy was developed in consultation with an academic librarian (DB), and using previously published search filters [27, 28]. Author (DB) conducted databases searches for studies from earliest record until January 312,017 that were available in: MEDLINE, MEDLINE in Process, EMBASE \& A + EDUCATION, PsycINFO, CINAHL, Cochrane Central Register of Controlled Trials (CENTRAL), ERIC, Proquest, Scopus. Search strategies were developed in MEDLINE and adapted according to the individual databases (see Additional file 1 for Medline search strategy). To identify additional articles the reference lists of all included studies were screened, lead authors of included studies were contacted, and hand searches of three key behavioural nutrition journals (Public Health Nutrition, International Journal of Behavioral Nutrition and Physical Activity and Health Education Research).

\section{Study selection}

Double independent searching for eligible studies by viewing titles and abstracts was conducted by authors $(\mathrm{NN}, \mathrm{LJ})$. The full texts of all potentially relevant studies were obtained and assessed against the inclusion criteria described above by authors in teams of two (NN, RS, MF, LJ). Disagreement regarding the eligibility of a study was resolved by discussion and consensus or consultation with a fifth author (CE). The number of articles at each screening stage is shown in Fig. 1.

\section{Data collection process}

Relevant information was extracted independently from included studies by two reviewers (NN, LJ), using a data extraction tool adapted from Cochrane data collection form for intervention reviews [29]. The following information was extracted: study aim, setting, country, study design, number randomized or allocated to treatment groups (for non-randomized trials), intervention components, duration and theoretical framework, primary outcomes, measures and results (mean and standard deviation (SD) data for all continuous outcomes) and information to assess risk of bias. Any discrepancies during data extraction were resolved through discussion and consensus or consultation with a third reviewer
(LW). Where key data were missing from the study reports, we attempted to contact the authors to obtain the information. Any information provided was incorporated into the review as appropriate.

\section{Risk of bias}

Risk of bias (extended criteria for cluster RCTs (C-RCTs)) for the included studies was assessed independently by two reviewers $(\mathrm{NN}, \mathrm{RH})$ using the Cochrane risk of bias tool [30]. Each study was assessed as being at 'high', 'low' or 'unclear' risk of bias for: sequence generation, allocation concealment, blinding of participants and personnel, blinding of outcome assessment, incomplete outcome data, selective outcome reporting and 'other' potential sources of bias, that is recruitment, loss of clusters, analysis and contamination. Any disagreement was resolved by discussion between the two reviewers.

\section{Data synthesis}

All dietary outcomes were assessed for suitability for pooled analysis with data from trials reporting a comparable outcome measure synthesised in meta-analyses. There were insufficient numbers of studies to pool data from non-randomised trial designs, or dichotomous trial outcomes. If insufficient data were reported to enable inclusion in meta-analysis (i.e. aggregate outcome fruit and vegetable consumption) authors were contacted for relevant information. Available data from the longest follow up period was extracted for synthesis in metaanalyses. We assessed C-RCTs for unit of analysis error. If analyses did not account for clustering of responses within settings, we used intra-class correlation coefficients (ICCs) from similar studies and outcomes to allow calculation of design effects and effective sample sizes to enable pooling in meta-analyses [30].

Where meta-analysis was possible, standardised mean differences (SMDs) were calculated to account for variable outcome measures for each comparison, using the generic inverse variance method, in a random-effect meta-analysis model via RevMan software. SMDs for each comparison were re-expressed as mean differences based on a familiar instrument from a study with the lowest risk of bias in that comparison (e.g. serves of vegetables or fruit provided), by multiplying the baseline standard deviation of the control group by the pooled SMD [30].

Heterogeneity was assessed using the $\mathrm{I}^{2}$ statistic. $\mathrm{I}^{2}$ values of lower than $50 \%$ were deemed to be acceptable levels of heterogeneity. For comparisons where $\mathrm{I}^{2}$ values were higher than $50 \%$, pre-specified subgroup analyses were conducted to investigate the source of heterogeneity by setting (centre-based care versus schools). Where pooling was not possible, findings were narratively synthesised according to primary and secondary outcomes. 


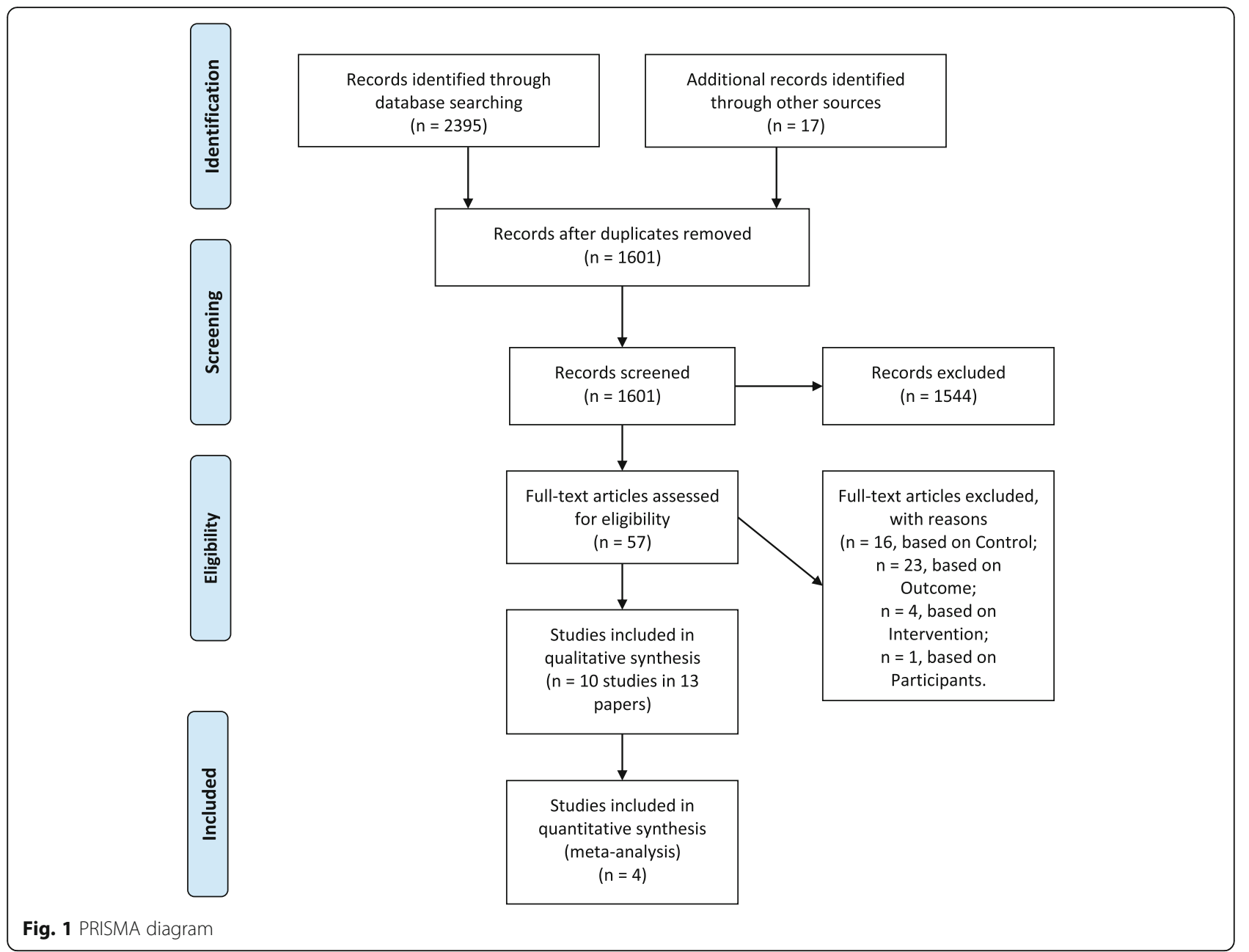

\section{Results}

\section{Study selection}

Overall 1601 records were screened for eligibility of which 1544 were excluded. Of the remaining 57 papers included in the full-text screen, 44 were excluded as they did not meet our eligibility criteria (see Fig. 1 for PRISMA diagram), leaving 13 papers, reporting on 10 trials included within this review.

\section{Study characteristics \\ Types of studies}

A description of the included studies is shown in Table 1. Of the 10 included trials, three were conducted in the U.S., [31-33] three in U.K., [21, 34-36] two in Australia $[37,38]$, one in Mexico [39] and one in Israel [40]. Eight of the studies employed cluster randomised-controlled trial designs, [21,31,32,34, 37-40] and two were conducted using quasi-experimental designs [24, 35].

\section{Participants}

Four trials were conducted in centre-based care $[24,32,37,38]$ with the number of participating centres ranging from six to 31 . These trials recruited samples of between 132 and 560 children with the age of participants ranging from 3 to 6 years. Of the six trials conducted in schools, $[21,31,34,35,39,40]$ the number of participating schools ranged from two to 89 . These trials recruited samples of between 238 and 2443 students with the age of participants ranging from 4 to 14 years.

\section{Interventions}

All 10 of the included trials utilised multicomponent interventions (employed two or more intervention strategies). All trials included intervention strategies to increase parent knowledge via delivery modes such as pamphlets, newsletters, posters or parent workshops. The majority of interventions (8/10) included an education component for children through videos, games, curriculum or activities [24, 31, 32, 34, 35, 37, 38, 40]. Four interventions, all school based, provided physical resources (e.g. lunch packs, containers) to support the packing of healthy lunchboxes [21, 31, 34, 35] while two provided incentives for children to taste vegetables and fruit [34-36]. Only two interventions, both centre-based 


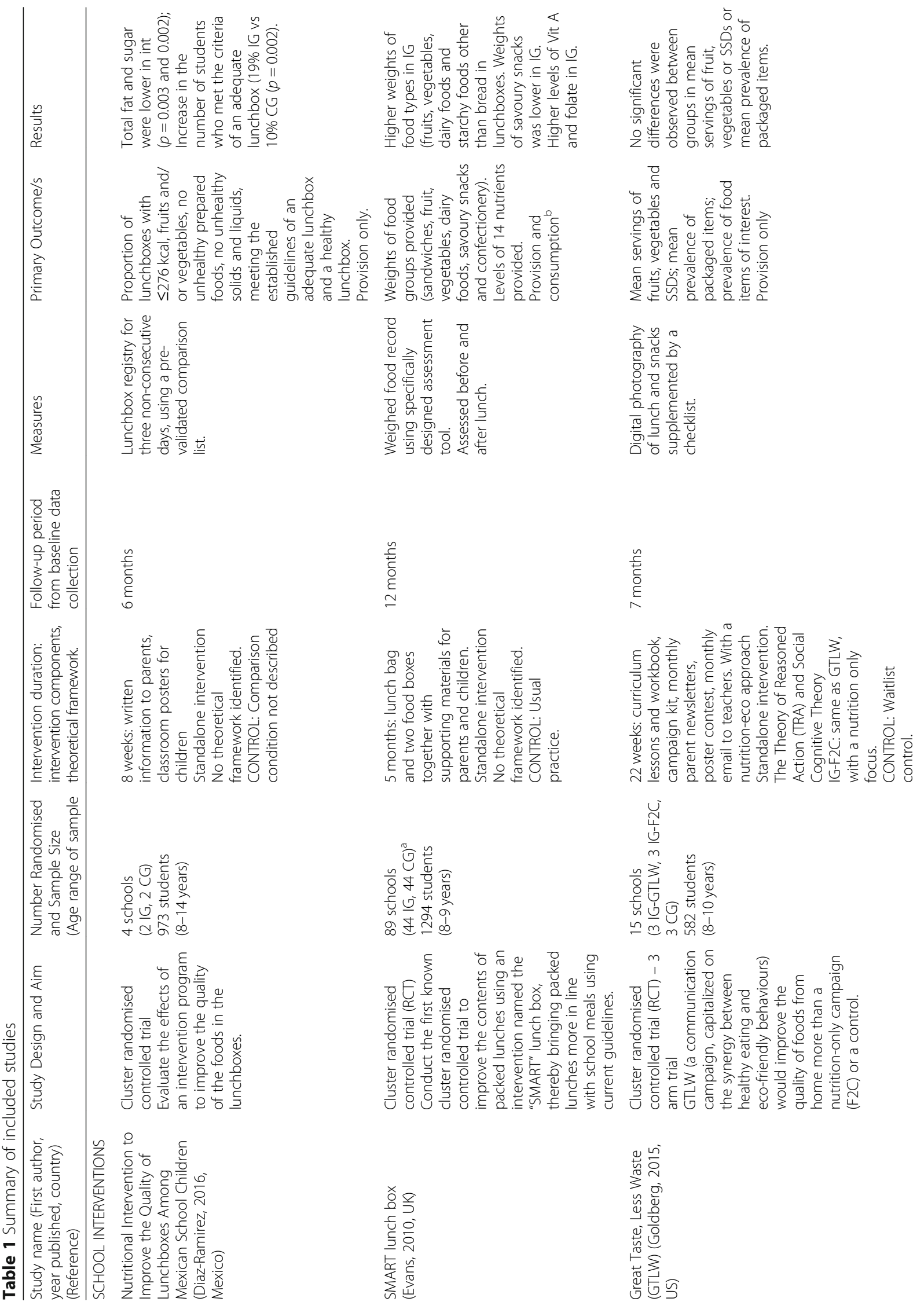




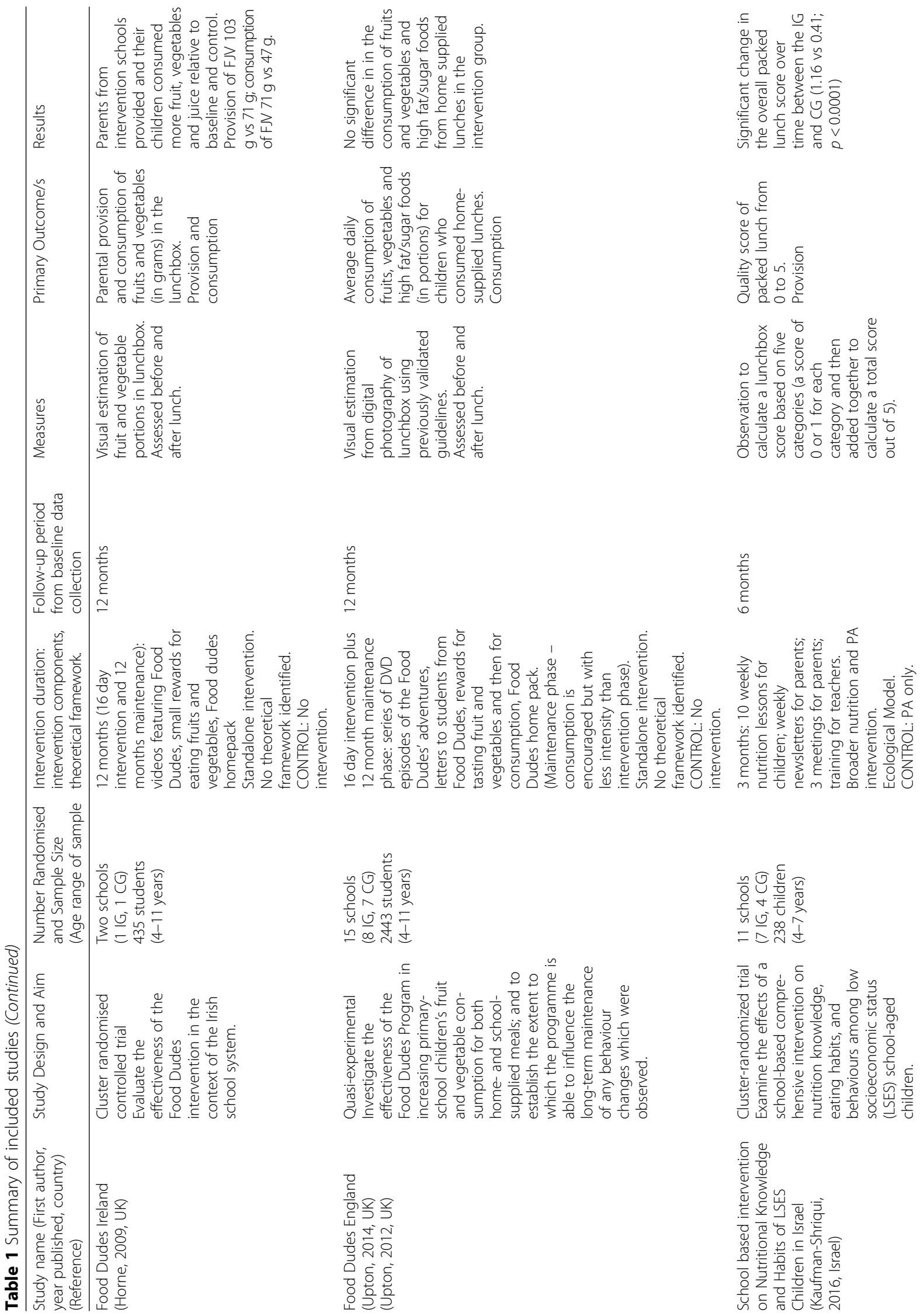




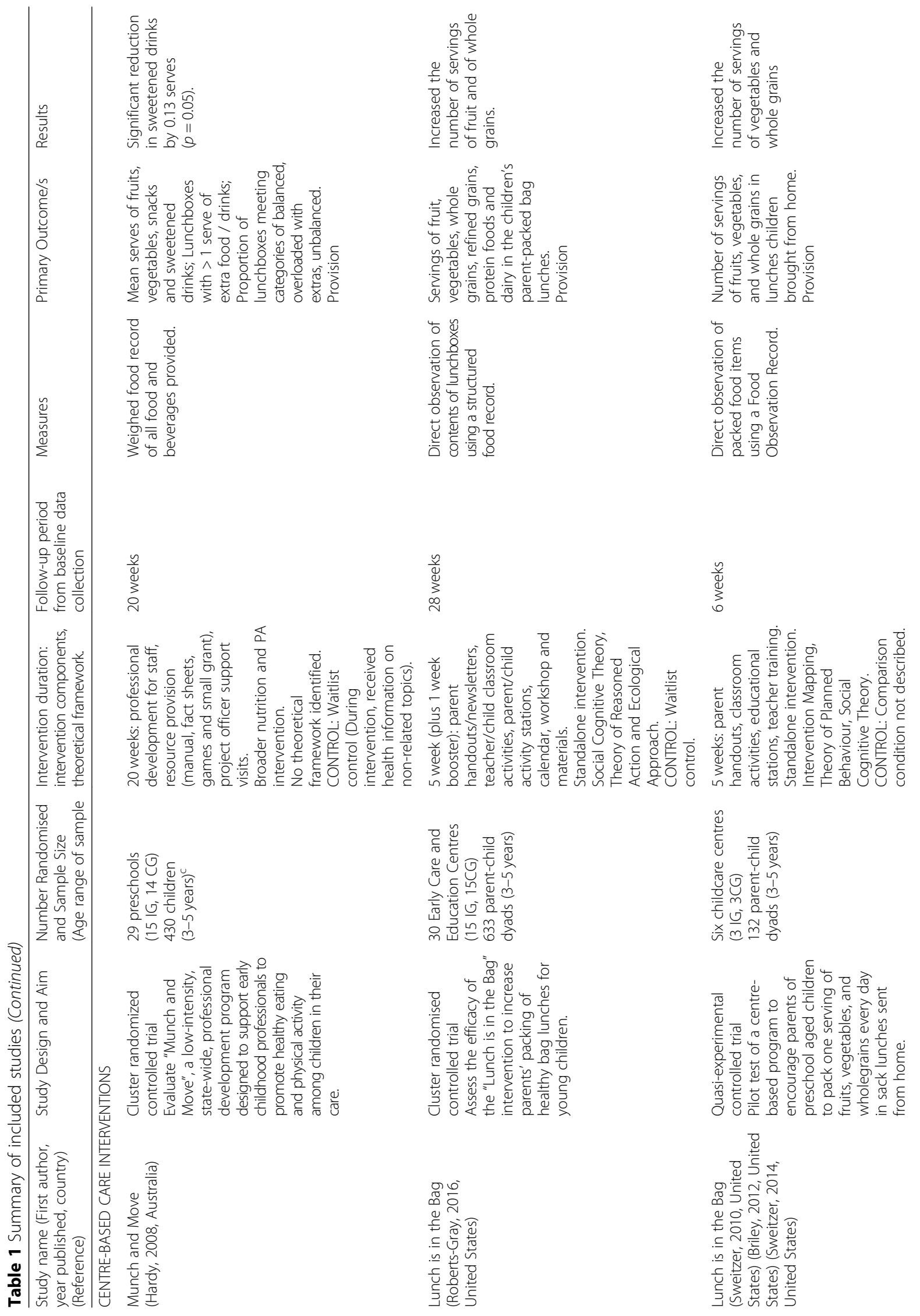




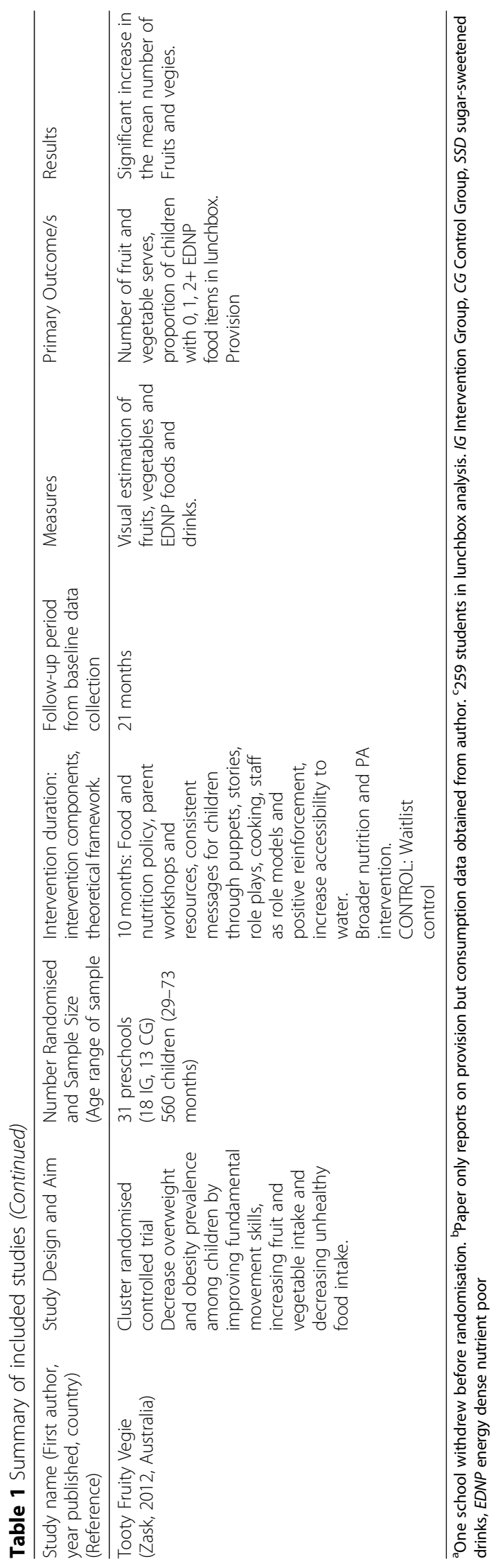


care studies, incorporated the development of a policy and the communication of this to parents [37, 38]. Seven of the studies were stand-alone lunchbox interventions $[21,24,31,32,34,35,39]$ whilst three were part of a larger child obesity prevention program, two occurring in centre-based care which included strategies to support centres to deliver physical activity (e.g. manuals, training, equipment) or information to parents targeting small screen recreation reduction [37, 38] and one in schools which included $15 \times 45$ min physical activity delivered to children by trained professionals [40]. Intervention duration across the studies ranged from five [24] to 8 weeks [39], 4 to 7 months, [31, 32, 37-40] and up to 12 months $[34,35]$ (see Table 1).

\section{Outcomes}

Follow-up data collection ranged from; 6 weeks post-baseline in one trial, [24] five to 7 months in five trials, [31, 32, 37, 39, 40] 12 months in three trials, [21, 34, 35] and 21 months in the remaining trial [38]. Of the eight studies that examined the impact of interventions on the nutritional content of foods and beverages provided from home [21, 24, $32,34,37]$ two studies reported on vegetables and fruit as a combined result, $[35,38]$ three reported on discretionary foods including snacks, or confectionary; $[21,37,38]$ three reported on sugar-sweetened drinks; [21,31,37]; and three reported on the proportion of lunchboxes meeting a pre-defined category that is "adequate/ healthy" [39],"balanced/ overloaded with extras/ unbalanced" [37] or a quality score for the packed lunch [40]. One study also reported on the mean weights of sandwiches, dairy foods, and other starchy foods provided [21].

Only three studies reported on the impact of the intervention on child dietary intake of packed foods $[21,34,36]$. Two reported on the mean weight in grams of vegetables and fruit individually consumed $[21,34,36]$ and one study [36] reported the combined serves and grams of vegetables and fruit consumed, and serves of high fat and sugar foods.

Lunchbox contents were measured via visual estimation $[24,32,34,38,40]$ or digital photography [31, 36] which some studies supplemented with a food observation checklist $[24,31,32]$ or a weighed measure [21, 37]. In one study [39] it was unclear what method was used. Children's dietary intake was measured by observing lunchboxes before and after school food breaks to see what was consumed [21, 34, 35]. Two studies, one an obesity prevention intervention in centre-based care [38] and one a nutrition and physical activity intervention in schools [40] included an objective measure of adiposity (BMI Z-score and mean waist circumference).

\section{Comparisons}

Two trials compared intervention strategies against no intervention, [34, 35] one against usual practice [21], one against a physical activity intervention only [40] and three against wait-list control, [32, 37, 38] of which one trial provided the control group general health information on an unrelated topic while on wait-list [37]. One trial included three trial arms, [31] i.e. two interventions (Great Taste Less Waste (GTLW) and Foods 2 Choose (F2C)) and one control, of which both intervention conditions sought to increase children's packing of vegetables and fruit. Both interventions were virtually identical however GTLW included "eco" messages that linked healthy eating to the environment. Two trials did not describe the comparison condition [24,39].

\section{Risk of bias}

Figures 2 and 3 summarize the risk of bias for each of the studies. Information related to random sequence generation was assessed as low in one study, [40] unclear in eight of the nine included studies [21, 24, 31, 32, 34, 37-39], and high risk for the other study as schools were purposefully selected to receive the intervention or act as control [35]. As allocation concealment was not possible in two studies they were assessed at high risk of bias [21,35] whereas the other eight were unclear. All of the studies were rated as being at high risk for performance bias given participants or personnel delivering the intervention were not blinded to intervention groups. Two studies reported that outcome assessors were blinded to group allocation and therefore were assessed at low risk of bias [37, 40], one study was high risk as data collectors undertaking outcome assessments were not blinded to group allocation [32], and unclear risk for the remaining seven studies. Four studies [21, 35, 37, 40] were rated as having high risk of bias due to incomplete outcome data due to large attrition (>20\%), two as low [31, 32] and unclear in the remaining four studies [24, 34, 38, 39]. Selective outcome reporting was rated as high risk of bias in two trials where not all primary (e.g. food groups and quantities of foods) [37] or secondary outcomes (e.g. BMI) [32] reported in the published study protocol or trial registration were included in the publication, low risk of bias in one study [40] and unclear risk of bias in the remaining seven trials. Seven studies were assessed as having high risk of other biases (related to not adequately describing if clustering was accounted for in analysis, [24, 34, 39] randomisation after baseline data collection, [31] or loss of clusters following randomisation. [21, 35, 40]), one study was rated at low risk of bias [37] and the remaining two were unclear [24, 38]. 


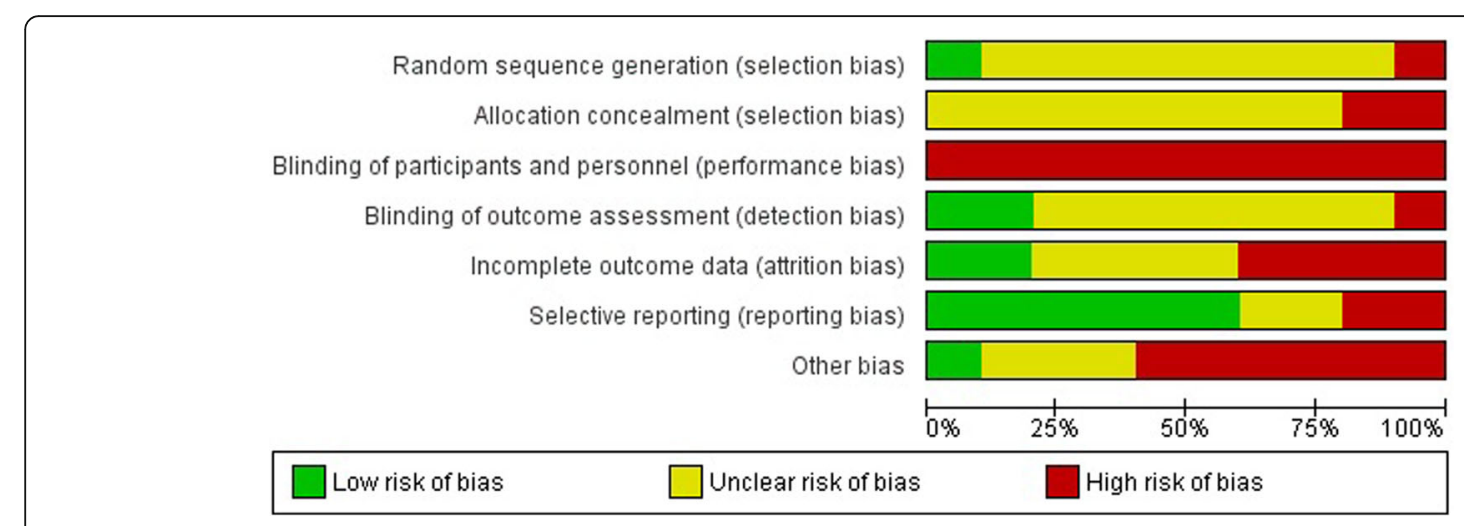

Fig. 2 Risk of bias graph

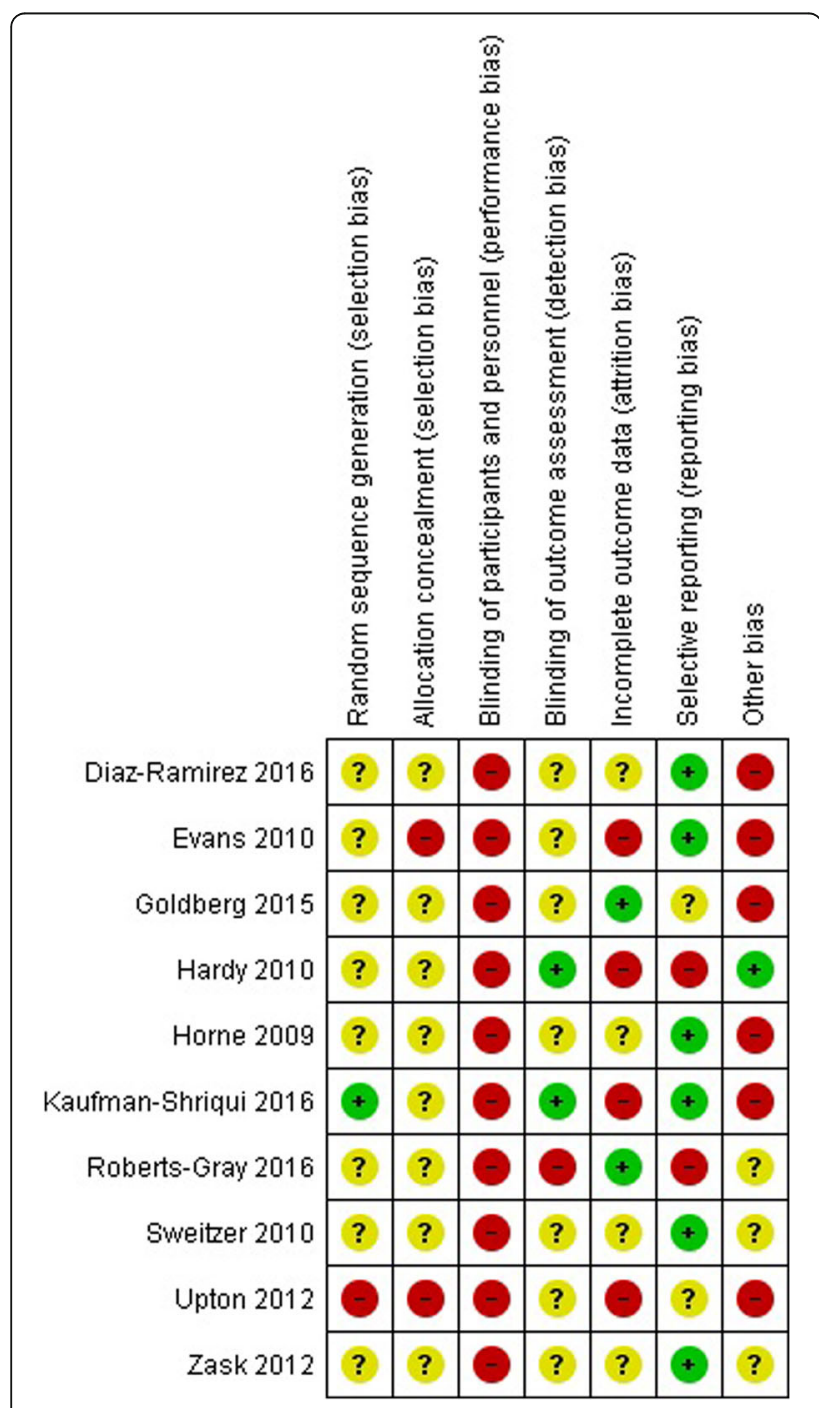

Fig. 3 Risk of bias summary

\section{Effects of interventions}

Impact of interventions on the nutritional content of foods and beverages provided from home (i.e. what is packed)

Fruit and vegetables: Meta-analyses of 2792 participants, from four studies (two in centre-base care [32, 37] and two in schools [21, 34]), revealed an overall significant increase in the provision of vegetables $(\mathrm{SMD}=0.4095 \%$ confidence interval (CI) 0.16 to $0.64, p=0.001, \mathrm{I}^{2}=82 \%$; equivalent to a mean difference of 0.28 serves) (Fig. 4) but not fruit $(\mathrm{SMD}=0.1695 \% \mathrm{CI}-0.04$ to $0.35, p=0.11$, $\mathrm{I}^{2}=71 \%$ ) (Fig. 5). Subgroup analyses were conducted on the basis of setting to investigate the source of heterogeneity. For the provision of vegetables, a overall significant increase remained in centre-based care $\left(\mathrm{SMD}=0.2695 \% \mathrm{CI} 0.08\right.$ to $0.44, p=0.005, \mathrm{I}^{2}=47 \%$; equivalent to a mean difference of 0.18 serves) but not schools $(\mathrm{SMD}=0.7295 \% \mathrm{CI}-0.22$ to $1.66, p=$ $0.13)$. For the provision of fruit, there was no overall effect in centre-based care $(\mathrm{SMD}=0.0495 \% \mathrm{CI}-0.10$ to $0.18, p=0.59)$ or schools $(\mathrm{SMD}=0.4295 \% \mathrm{CI}$ -0.21 to $1.04, p=0.19$ ).

The other four non-randomised trials that measured the packing of vegetables or fruit were not included in the meta-analysis. Two quasi-experimental trials, one conducted in Australian pre-schools [38] and one in U.S. pre-schools, [24] reported significant effects of the intervention on the provision of vegetables and fruit. The Australian study reported an improvement in the number of vegetables and fruits provided (combined) (mean difference $=0.61$ serves, standard error (SE) $=0.14, p=0.001)$ whilst the U.S. study reported a significant increase in mean number of servings of vegetables 0.344 ( $\mathrm{SE}=0.100$, p 0.001) but not fruit (mean serving $0.065, \mathrm{SE}=0.124, \mathrm{p} 0.600$ ). Similarly, a trial in two schools in Ireland, reported that children in the experimental school were provided with significantly more fruit, vegetable and juice in their lunchboxes than control children $(\mathrm{t}=3.5, p<0.001)$ [34]. Another trial undertaken in Mexican schools reported a no significant effect 


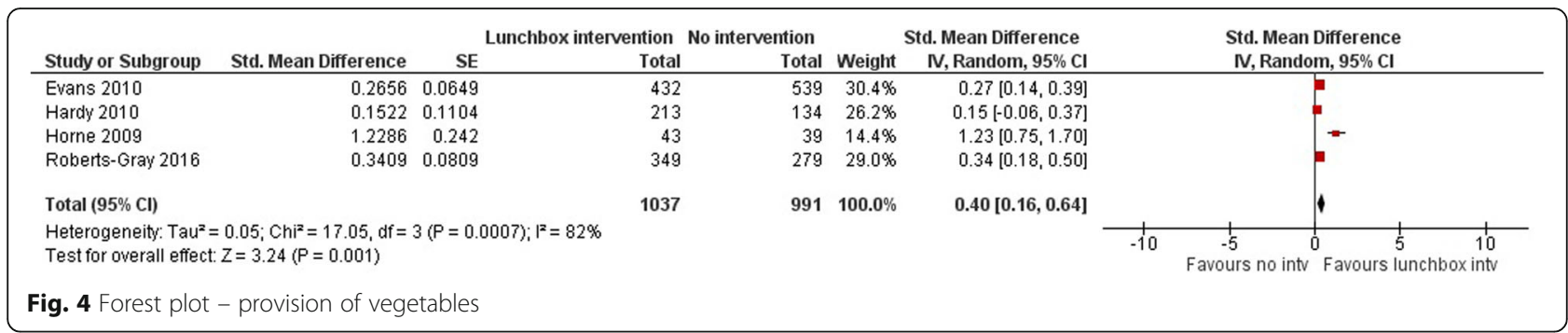

on the vegetable and fruit content of lunchboxes of intervention vs control students $(39.0 \%$ vs $37.0 \% p=$ 0.57) [39].

Discretionary foods including snacks, confectionary Four studies reported on the impact on the provision of discretionary foods [21, 32, 37, 38]. Of those, one C-RCT, conducted in centre-based care as part of a broader child obesity prevention program, reported significant effects of the intervention on the proportion of children that had lunchboxes with no energy-dense nutrient-poor food items (difference at follow-up 29.1\%; SE not able to be calculated, $p<0.001$ ) [38]. Conversely, the other study in centre based care, also delivered as part of a broader child obesity prevention program, found no significant impact on lunchboxes containing serves of snacks (mean difference $=0.06(95 \%$ CI -0.34 to 0.46$) p=0.75$ ); or one or more serves of extra foods (odds ratio $=0.90(95 \%$ CI 0.48 to 1.70) $p=0.74)$ [37]. One school-based intervention reported mixed results, with a significant weight reduction in the provision of savoury snacks i.e. grams of crisps and other salted snacks (mean difference $-2.895 \%$ CI -5.5 to $-0.2 p=$ 0.04 ) but no reported change in the weight of confectionary (foods containing chocolates, cereal bars and sweets) provided (mean difference-2.1 95\% CI -5.6 to 1.5 $p=0.26$ [21]. The other school based study, also reported mixed results, with no impact on the provision of chips but found a significant impact on the servings of sweets (fruit drinks, cookies and candy) (mean difference -0.43 servings, $\mathrm{SE}=0.11, p<0.001$ ) [32].

Sugar-sweetened drinks- One study in centre-based care reported significant reductions in the provision of sugar-sweetened drinks by 0.13 serves (approximately $46 \mathrm{mls}$; $95 \%$ CI -0.27 to 0.002 ; $(p=0.05)$ ) [37] while two studies in schools reported no significant change in weight of sugar-sweetened drinks provided (mean difference $=-5.0(95 \% \mathrm{CI}-34.3$ to $24.4 \mathrm{p}=0.74)$ [21] or mean servings of sugar-sweetened drinks provided [31] (intervention vs control $p=0.98$; intervention vs nutrition only $p=0.80$ ).

"Healthy" lunchboxes- Three studies used predefined categories to measure the impact of interventions on the contents of children's lunchboxes [37, 39, 40]. One study in centre-based care [37] reported no significant change in the provision of "balanced" lunchboxes (that is containing at least a sandwich or home cooked meal and either fruit or vegetables, with the allowance of one extra serve of food or beverage) (difference at follow-up $=0.85$ (95\% CI 0.35 to 2.25) $p=0.72$ ). One study in schools [39] reported a significant difference in the proportion of intervention students that had an adequate lunchbox vs. controls $(19.1 \%$ vs $9.6 \% p=0.002)$ (that is containing $<276 \mathrm{cal}$, vegetables and/or fruits and an item prepared at home) and, although the relative effect size was large there was no significant difference for those that had a healthy lunchbox (that is containing vegetables and/or fruits, water and no unhealthy foods) $(10.2 \%$ vs $5.9 \% p=$ 0.09 ). Whilst the remaining study, also in schools, reported a significant improvement in the packed lunch score at follow-up between intervention and control $(1.16 \pm 0.16,120 \%$ vs $0.41 \pm 0.18,42 \% p=<0.001)$ [40]

Other foods (including sandwiches, dairy foods, whole grains)- Three studies, one school-based study [21] and two centre-based care studies [24, 32] examined the

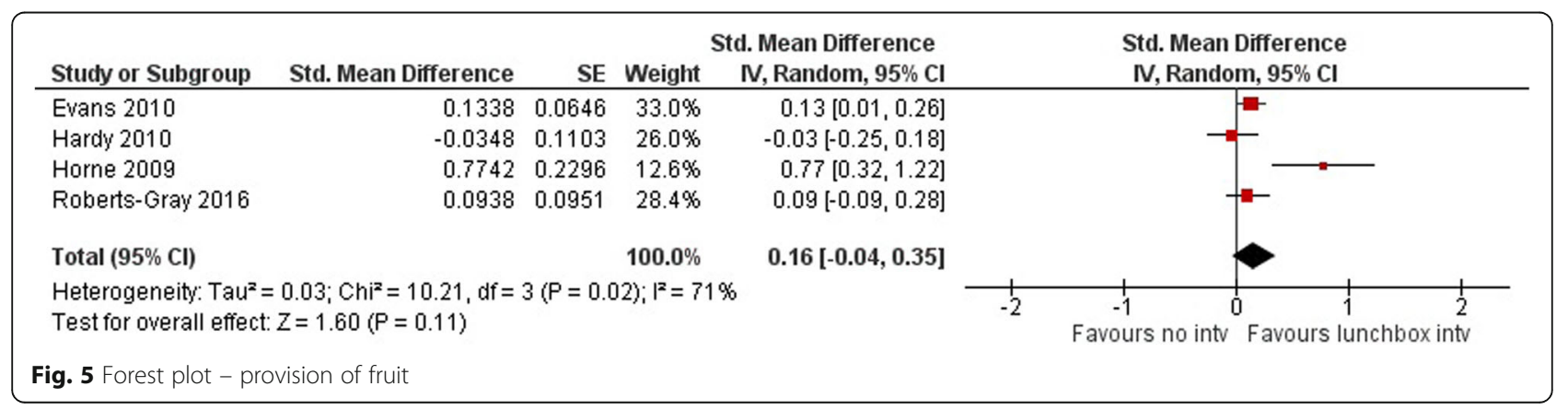


impact on the provision of other core foods. The school-based study reported a significant difference in the mean weight of dairy food i.e. cheese and milk based desserts (mean difference $8.4 \mathrm{~g}$ 95\% CI $2.0 \mathrm{~g}$ to $14.9 \mathrm{~g}$ $p=0.01$ ) and starchy foods other than bread (mean difference $7.4 \mathrm{~g} 95 \% \mathrm{CI} 0.5 \mathrm{~g}$ to $14 . \mathrm{g} 4 p=0.04$ ) provided to intervention children compared to control. However no difference was found in the mean weight of sandwiches (mean difference $3.2 \mathrm{~g}$; 95\% CI -4.7gto $11.1 \mathrm{~g} ; p=0.43$ ) and unsweetened drinks (fruit juice and milk) provided (mean difference $=13.6 \mathrm{~g} ; 95 \% \mathrm{CI}-17.2 \mathrm{~g}$ to $44.3 \mathrm{~g} ; p=$ 0.39) [21]. The two studies in centre-based care, examined the impact on the provision of wholegrains, with both studies showing an increase in the mean number of servings of wholegrains packed compared to control (0.34 servings; $\mathrm{SE}=0.13 ; p=0.009)[24,41]$ and (0.49 servings; $\mathrm{SE}=0.15 ; p=0.001$ ) [32]. In addition, the study by Roberts-Gray, [32] found no impact on the provision of protein-based foods (e.g. meats, nuts, etc.) but showed an increase in the servings of dairy which the authors attributed to a decrease in provision in control centres, not an increase in intervention centres $(0.14$ servings; $\mathrm{SE}=0.05$; $p=0.011$.

\section{Impact of interventions on child dietary intake}

Three school-based studies reported on the impact of interventions on children's dietary intake of vegetables and fruit [21,34-36] one being the pilot [34] for a larger trial [35]. The pilot study by Horne et al. [34] reported a significantly higher mean consumption of fruit, vegetable and juice among intervention students compared to control $(\mathrm{t}=3.7, p>0.001)$ [34]. Conversely, the larger trial by Upton et al., [35] found no change in fruit and vegetables consumed by intervention students at 3-months compared to baseline, however a non-significant decrease at 12 months compared to baseline $(d=-0.16$, $95 \%$ CI $-0.30,0.01)$. In the control schools however child fruit and vegetable consumption was significantly higher at 3 months compared to baseline $(\mathrm{d}=0.26,95 \% \mathrm{CI}$ $-0.12,0.38)$ but not at 12 months $(\mathrm{d}=0.05,95 \% \mathrm{CI}$ $-0.08,0.16)$. The study by Evans et al. [21] found a significant improvement in the consumption of vegetables $(6.2 \mathrm{~g}, \mathrm{SE}=1.8 \mathrm{~g}, 95 \%$ CI $2.7-9.8 \mathrm{~g}$ ) but not fruit $(9.0 \mathrm{~g}$, $\mathrm{SE}=5.7 \mathrm{~g}, 95 \% \mathrm{CI}-2.5$ to $20.4 \mathrm{~g}$ ). The school based study by Upton et al., [36] also reported on the impact of the intervention on child consumption of high fat $(>17.5 \mathrm{~g}$ of total fat per $100 \mathrm{~g})$ and high sugar $(>22.5 \mathrm{~g}$ of total sugars per $100 \mathrm{~g}$ ) foods and found no intervention effect between groups.

\section{Secondary outcome i: impact of interventions on child adiposity (weight or BMI)}

One study, [38] an obesity prevention intervention in centre-based care that included a range of strategies in addition to those targeting the content of student lunchboxes, found a significant adjusted difference on BMI $\mathrm{z}$-score $(-0.15, \mathrm{SE}=0.07, p=0.022)$ and waist circumference $(-0.80 \mathrm{~cm}, \mathrm{SE}=0.35, p=0.020)$ among children attending intervention services relative to control. However the intervention had no overall significant effect on overweight and obesity prevalence $(12.5 \%$ among control vs $11.5 \%$ amount intervention at follow-up). Similarly, a nutrition and physical activity intervention in schools [40] reported a reduction in 0.1 points in BMI $\mathrm{z}$-score in both intervention and control arms, which they attributed to both intervention and control receiving the physical activity intervention.

\section{Discussion}

This review is the first to report on the effectiveness of lunchbox interventions aiming to improve the foods and beverages packed and consumed by children attending centre-based care or school and subsequent impact on adiposity. Data pooled from four of the 10 included trials suggest that to date interventions have had a modest impact on the provision of vegetables in children's lunchboxes. Evidence for the effectiveness of interventions on what is packed in children's lunchboxes in relation to discretionary foods, sugar-sweetened drinks or other core foods were equivocal. Similarly, of the few trials reported the effects of interventions on child dietary intake and adiposity findings were mixed. Such findings suggests that interventions targeting the foods packed for child consumption at school or childcare services can have positive effects, however future research is warranted.

Interestingly, meta-analysis results identified improvements in the contents of children's lunchboxes related to the provision of vegetables, but not fruit. There was also evidence that improved provision of vegetables packed for children also led to increased vegetable consumption. Given the small proportion of children that meet recommended daily serves of vegetables these findings are encouraging as studies report child dietary behaviours are determined, in part, by the availability and exposure to heathy foods $[7,42]$. The findings, however, are in contrast to the findings of reviews of school based vegetable and fruit programmes that typically report greater effects on fruit rather than vegetable intake [43]. Previous programmes to improve vegetable and fruit intake tend to focus on fruit provided as a snack. Potentially, interventions targeting child lunchboxes may provide greater opportunity to increase vegetable intake through modifying snack and meal (lunch) eating occasions. While the effects of vegetable provision were reported, fruit was more frequently packed and consumed by children in the included trials thus potentially explaining the null effect. It does therefore suggest more comprehensive 
approaches to improve vegetable intake are warranted, such as enhancing the school environment to promote children's liking and expectation of vegetable consumption at lunchtime.

The findings for the provision of discretionary foods, sugar-sweetened drinks and other core foods were less consistent. These results suggest that removing less healthy items from children's lunchboxes, such as sugary drinks and sweet and savoury snacks, may be more challenging. As parents are primarily responsible for the packing of lunchboxes of children, greater formative evaluation with parents regarding the barriers to removing such foods may be required to improve the development and effectiveness of future interventions targeting discretionary foods. This may include addressing parents' concerns regarding time, cost or food safety [44]. Additionally, improvements to the availability of healthy foods in other environments including supermarkets, quick service restaurants and sporting clubs as well as to the promotion and marketing of food to children may be required to improve child intake of these foods. For example, progress has been made in the UK on reducing portion sizes of sweet and savoury snacks as part of the childhood obesity strategy [45].

This review identified a number of opportunities to strengthen the effects of existing interventions, particularly in relation to the packing of discretionary foods. While all included studies in the review incorporated a parent component, many simply relied on passive information dissemination strategies which are typically reported to have limited reach and are inadequate to change behaviour [46]. The use of active intervention strategies with the capacity to deliver a variety of evidence based behavioural change strategies (e.g. electronic based interventions) may be more likely to enhance intervention effects. Furthermore, setting-based interventions that undertake a comprehensive approach to improving child health as encouraged by such frameworks as the health promoting schools framework may improve the impact of interventions. For example, trials examining lunchbox interventions supported by explicit school policies and related teaching curriculum appear warranted.

There are a number of strengths to this review. A comprehensive search strategy, utilising robust review methods was undertaken and where possible the estimates of intervention effects were quantified by using meta-analysis adjusting for clustering of children within centre-based care and schools where necessary. However, a number of limitations are worth considering when interpreting the review findings. Only studies published in English were included, which may have excluded other efficacious studies. A number of the characteristics of included studies were consistently assessed as at high risk of bias. Of concern was the lack of random sequence generation and blinding of outcome assessors, which may reduce the confidence of the individual studies trial findings. The longest follow-up period within each study was used in the meta-analysis, however, it is possible that intervention effects may have attenuated in studies with longer follow-up periods than those with shorter follow-up periods. Furthermore, as most of the trials had follow-up periods of less than 12-months it is not possible to know if intervention effects were sustained over longer periods of time. High levels of heterogeneity were also evident from forest plots. The source of heterogeneity was unclear and could be due to differences in population, intervention, outcome or other methodological factors including those not reported in the included trials. The small number of eligible trials precluded examination of heterogeneity by these factors. The meta-analysis is limited by having only a small number of studies, all of which were multi-component interventions, with various outcomes of interest. Whilst subgroup analysis is included, the heterogeneity of these studies should be considered when interpreting these findings. The external validity of the review findings may also be limited given different school food environments internationally. For example, it is also possible that in countries where approximately half of children have a school meal and half take in a packed lunch (such as the UK) specific types of intervention programmes would have a different impact compared to the same intervention introduced in a country where generally no school meal is provided (such as Australia). More trials are needed to confirm these findings. Despite these limitations, given the foods that children bring from home to eat at centre-based care or school, contribute significantly to their daily energy intake, interventions targeting children's lunchboxes remain promising as a strategy to improve child public health nutrition.

\section{Conclusion}

Interventions to improve the diet quality of packed lunches in children have a modest impact in improving provision of vegetables. However, improvements in other foods such as sugary drinks and other sweet and savoury snacks are not consistent between studies. Given the significant influence parents and caregivers have on the contents of children's lunchboxes, interventions should continue to engage parents through active intervention strategies and report the reach of such strategies. Future policies related to children's packed lunches should aim to further increase the success of improving packed lunches through improvements in the food environment such as increasing the availability of healthy foods and reductions in the marketing of sweet foods and drinks 
and savoury snacks. The potential concern of any public health intervention is widening health disparities. Future lunchbox interventions should aim to report on intervention effects by socio-economic status of children to ensure this is avoided. Given the limited number of studies that assessed child adiposity future more robust trials are required to investigate the potential impact of lunchbox interventions on child diet, weight and BMI.

\section{Additional file}

Additional file 1: Medline search strategy. (DOCX $15 \mathrm{~kb}$ )

\section{Abbreviations}

BMI: Body Mass Index; GRADE: Grading of Recommendations, Assessment, Development and Evaluations; PRISMA: Preferred Reporting Items for Systematic Reviews and Meta-Analyses; RCT: Randomised Controlled Trials; U.K.: United Kingdom; U.S.: United States

\section{Acknowledgements}

The authors wish to thank Clare Desmet for assistance with the review. The work was supported by infrastructure support from the Hunter Medical Research Institute (HMRI) and Hunter New England Population Health. NN was supported by a National Health and Medical Research Council (NHMRC) Translating Research Into Practice (TRIP) Fellowship and a Hunter New England Clinical Research Fellowship; RS was supported by a National Health and Medical Research Council (NHMRC) Translating Research Into Practice (TRIP) Fellowship, LW was supported by a National Health Medical Research Council Career Development Fellowship and a Heart Foundation Future Leader Fellowship and a Hunter New England Clinical Research Fellowship; SY was supported by a Heart Foundation Postdoctoral Fellowship. MF was supported by a Hunter New England Clinical Research Fellowship.

\section{Funding}

None to report

\section{Availability of data and materials}

The dataset supporting the conclusions of this article is included within the article (and its additional files).

\section{Authors' contributions \\ Contributions of authors: NN, RS and LW conceived and designed the study. DB and NN developed the search strategy and DB conducted the search. $N N, L J, R S$ and MF screened studies for inclusion with CE acting as third reviewer for all stages; $\mathrm{NN}$ and $\mathrm{LJ}$ extracted data and $\mathrm{NN}$ and $\mathrm{RH}$ conducted ROB. CE and RH conducted meta-analysis. NN drafted the manuscript with all co-authors contributing to drafts of the paper. All authors approved the final manuscript.}

\section{Ethics approval and consent to participate}

Not applicable

\section{Consent for publication}

This manuscript has not been published - either in print or electronic form or is being considered for publication elsewhere. All authors approved the final version for submission.

\section{Competing interests}

Author CE has received funding from Unilever UK to repeat a survey of children's packed lunches in England in 2016.

\section{Publisher's Note}

Springer Nature remains neutral with regard to jurisdictional claims in published maps and institutional affiliations.

\section{Author details}

Hunter New England Population Health, Hunter New England Local Health District, Newcastle, Australia. ${ }^{2}$ School of Medicine and Public Health, The University of Newcastle, Newcastle, Australia. ${ }^{3}$ Priority Research Centre for Health Behaviour, The University of Newcastle, Newcastle, Australia. ${ }^{4}$ Hunter Medical Research Institute, Newcastle, Australia. ${ }^{5}$ University Library, Academic Division, University of Newcastle Australia, Newcastle, Australia. ${ }^{6}$ Nutritional Epidemiology Group, School of Food Science and Nutrition, University of Leeds, Newcastle, UK. ${ }^{7}$ Hunter New England Population Health, Locked Bag No. 10, Wallsend, NSW 2287, Australia.

Received: 27 June 2018 Accepted: 10 April 2019

Published online: 29 April 2019

\section{References}

1. Hardy L, et al. NSW schools physical activity and nutrition survey (SPANS) 2010: full report. Sydney: Ministry of Health; 2011.

2. Commonwealth Scientific Industrial Research Organisation (CSIRO), 2007 Australian National Children's Nutrition and Physical Activity Survey - Main Findings, Department of Health and Ageing, Editor. 2008, Commonwealth of Australia: Canberra.

3. Nicklas T, Johnson R. Position of the American dietetic association: dietary guidance for healthy children ages 2 to 11 years. J Am Diet Assoc. 2004; 104(4):660-77.

4. Whitrow MJ, et al. Core food intakes of Australian children aged 9-10 years: nutrients, daily servings and diet quality in a community cross-sectional sample. J Hum Nutr Diet. 2016;29(4):449-57.

5. Krebs-Smith $\mathrm{SM}$, et al. Americans do not meet federal dietary recommendations. J Nutr. 2010;140(10):1832-8.

6. National Fruit and vegetable Alliance, National Action Plan to Promote Health Through Increased Fruit and Vegetable Consumption 2010 Report Card, produce for better Health Foundation, editor. 2010, https://nfva.org/ national_action_plan.html. Accessed 21 Sept 2017.

7. Rasmussen $M$, et al. Determinants of fruit and vegetable consumption among children and adolescents: a review of the literature. Part I: quantitative studies. Int J Behav Nutr Phys Act. 2006;3(22):19.

8. Hardy LL, M.S., Drayton BA, Bauman, A., NSW Schools Physical Activity and Nutrition Survey (SPANS) 2015: Full Report. 2016, NSW Department of Health: Sydney.

9. Public Health England, National Diet and Nutrition Survey. Results from Years 5-6 (combined) of the Rolling Programme (2012/13-2013/14). 2016: London.

10. Venn AJ, et al. Overweight and obesity from childhood to adulthood: a follow-up of participants in the 1985 Australian schools health and fitness survey. Med J Aust. 2007;186(9):458-60.

11. World Health Organisation. Population-based approaches to childhood obesity prevention. Geneva: WHO Press, World Health Organization; 2012. https://apps.who.int/iris/bitstream/handle/10665/80149/9789241504782_ eng.pdf; jsessionid=40C77975EF84D9589F5B409160ED6638? sequence $=1$.

12. Wolfenden $\mathrm{L}$, et al. Strategies to improve the implementation of healthy eating, physical activity and obesity prevention policies, practices or programmes within childcare services. Cochrane Database of Systematic Reviews, 2016(10)

13. Wolfenden L, Nathan NK, Sutherland R, Yoong S, Hodder RK, Wyse RJ, Delaney T, Grady A, Fielding A, Tzelepis F, Clinton-McHarg T, Parmenter B, Butler P, Wiggers J, Bauman A, Milat A, Booth D, Williams C. Strategies for enhancing the implementation of school-based policies or practices targeting risk factors for chronic disease. . Cochrane Database Syst Rev, 2017. Issue 11(Art. No.: CD011677).

14. Sanigorski AM, et al. Lunchbox contents of Australian school children: room for improvement. Eur J Clin Nutr. 2005:59(11):1310-6.

15. Radcliffe BC, Cameron CV, Baade PD. Nutrient intakes for young children: implications for long-day child-care nutrition recommendations. Nutr Diet. 2002:59(3):187-90.

16. Farris AR, et al. Nutritional comparison of packed and school lunches in prekindergarten and kindergarten children following the implementation of the 2012-2013 National School Lunch Program standards. J Nutr Educ Behav 2014:46(6):621-6.

17. Evans $C$, et al. A cross-sectional survey of children's packed lunches in the UK: food-and nutrient-based results. J Epidemiol Community Health. 2010 https://doi.org/10.1136/jech.2008.085977. 
18. Bell AC, Swinburn BA. What are the key food groups to target for preventing obesity and improving nutrition in schools? Eur J Clin Nutr. 2004;58(2):258-63.

19. Dresler-Hawke E, Whitehead D, Parker L. Children's selection of fruit and vegetables in a 'dream versus healthy'lunch-box survey. Health Educ J. 2012; 71(6):736-45.

20. Perez-Rodriguez $M$, et al. Children's lunch box after the implementation of a guideline to regulate the foods and beverages for sale in schools in Mexico City. FASEB J. 2012;26(1 Supplement):33.5.

21. Evans $C E$, et al. SMART lunch box intervention to improve the food and nutrient content of children's packed lunches: UK wide cluster randomised controlled trial. J Epidemiol Community Health. 2010;64(11):970-6.

22. Lesley D. Contribution of "packed lunches" to the dietary intake of 11-12year-old children. Nutrition \& Food Science. 1999;99(4):181-6.

23. Jones J, et al. Dietary intake and physical activity levels of children attending Australian childcare services. Nutrition \& Dietetics. 2017;74(5):446-53.

24. Sweitzer SJ, et al. Lunch is in the bag: increasing fruits, vegetables, and whole grains in sack lunches of preschool-aged children. J Am Diet Assoc. 2010;110(7):1058-64

25. Santillana Marin E, Jimenez-Cruz A, Bacardi-Gascon M. Programs aimed to increase the nutritional content of lunch packs; systematic review. Nutr Hosp. 2013;28(6):1802-5.

26. Moher $\mathrm{D}$, et al. Preferred reporting items for systematic reviews and metaanalyses: the PRISMA statement. Ann Intern Med. 2009;151(4):264-9 W64.

27. Seward $\mathrm{K}$, et al. Factors that influence the implementation of dietary guidelines regarding food provision in Centre based childcare services: a systematic review. Prev Med. 2017;105:197-205.

28. Nathan N, et al. Barriers and facilitators to the implementation of physical activity policies in schools: a systematic review. Prev Med. 2017;107:45-53.

29. The Cochrane Collaboration, Data collection form for intervention reviews for RCTs only [internet]. p. 2015. Available from: https://handbook-5-1. cochrane.org/chapter_7/table_7_3_a_checklist_of_items_to_consider_in_ data_collection.htm. [cited 2015 September 11].

30. Higgins JPT and G. S., Cochrane Handbook for Systematic Reviews of Interventions Version 5.1.0 [updated March 2011]. . 2011, The Cochrane Collaboration.

31. Goldberg JP, et al. Great taste, less waste: a cluster-randomized trial using a communications campaign to improve the quality of foods brought from home to school by elementary school children. Prev Med. 2015;74:103-10.

32. Roberts-Gray C, et al. Efficacy of the lunch is in the bag intervention to increase parents' packing of healthy bag lunches for young children: a cluster-randomized trial in early care and education centers. Int J Behav Nutr Phys Act. 2016;13:3.

33. Sweitzer SJ, et al. Psychosocial outcomes of lunch is in the bag, a parent program for packing healthful lunches for preschool children. J Nutr Educ Behav. 2011;43:536-42.

34. Horne PJ, et al. Increasing parental provision and children's consumption of lunchbox fruit and vegetables in Ireland: the food dudes intervention. Eur J Clin Nutr. 2009;63(5):613-8.

35. Upton D, Upton P, Taylor C. Increasing children's lunchtime consumption of fruit and vegetables: an evaluation of the food dudes programme. Public Health Nutr. 2013;16(6):1066-72.

36. Upton P, Taylor C, Upton D. The effects of the food dudes Programme on children's intake of unhealthy foods at lunchtime. Perspect Public Health. 2015;135(3):152-9.

37. Hardy $L L$, et al. Munch and move: evaluation of a preschool healthy eating and movement skill program. Int J Behav Nutr Phys Act. 2010;7:80.

38. Zask A, et al. Tooty fruity vegie: an obesity prevention intervention evaluation in Australian preschools. Health Promot J Austr. 2012;23(1):10-5.

39. Diaz-Ramirez G, Jimenez-Cruz A, Bacardi-Gascon M. Nutritional intervention to improve the quality of lunchboxes among Mexican school children. J Community Health. 2016;41(6):1217-22.

40. Kaufman-Shriqui $V$, et al. Effect of a school-based intervention on nutritional knowledge and habits of low-socioeconomic school children in Israel: a cluster-randomized controlled trial. Nutrients. 2016;8(4):234.

41. Briley ME, et al. Unbundling outcomes of a multilevel intervention to increase fruit, vegetables and whole grains parents pack for their preschool children in sack lunches. Am J Health Educ. 2012;43(3):135-42 8p.

42. Wyse R, Wolfenden L, Bisquera A. Characteristics of the home food environment that mediate immediate and sustained increases in child fruit and vegetable consumption: mediation analysis from the healthy habits cluster randomised controlled trial. Int J Behav Nutr Phys Act. 2015;12(1):118.

43. Evans CEL, et al. Systematic review and meta-analysis of school-based interventions to improve daily fruit and vegetable intake in children aged 5 to 12 y. Am J Clin Nutr. 2012;96(4):889-901.

44. Hawthorne DL, et al. Parental reports of lunch-packing Behaviours lack accuracy: reported barriers and facilitators to packing school lunches. Can J Diet Pract Res. 2018;79(3):99-105.

45. Rimmer A. Industry must cut calories in savoury food products by $20 \%$, says Public Health England. BMJ. 2018;360:k1067. https://doi.org/10.1136/bmj. k1067.

46. Hingle MD, et al. Parental involvement in interventions to improve child dietary intake: a systematic review. Prev Med. 2010;51(2):103-11.

\section{Ready to submit your research? Choose BMC and benefit from:}

- fast, convenient online submission

- thorough peer review by experienced researchers in your field

- rapid publication on acceptance

- support for research data, including large and complex data types

- gold Open Access which fosters wider collaboration and increased citations

- maximum visibility for your research: over $100 \mathrm{M}$ website views per year

At BMC, research is always in progress.

Learn more biomedcentral.com/submissions 